\title{
Platelet safety range before splenectomy for hypersplenism : based on 244 cases of splenectomy in hepatolenticular degeneration patients
}

\author{
S.T. Wang ${ }^{1}$, H. Feng ${ }^{* 2,3}$, H. Peng ${ }^{* 3,4}$, L. Huang ${ }^{2,3}$, F.H. Zhou ${ }^{2,3}$, Q.S. Yu ${ }^{2,3}$ \\ (1) The First Affiliated Hospital of University of science and technology of China, Hefei, China (2) ; The First Affiliated Hospital of Anhui University of Chinese Medicine, \\ Hefei, China ; (3)Department of surgery, anhui academy of traditional Chinese medicine, Hefei, China ;(4) Anhui university of traditional Chinese medicine, Hefei, China.
}

\begin{abstract}
Background and study aims: To investigate the safety and efficacy of splenectomy for hepatolenticular degeneration (HLD) patients with PLT less than $20 \times 10^{\circ} / \mathrm{L}$.

Patients and methods : A total of 244 HLD patients with hypersplenism underwent splenectomy. According to the preopera-tive PLT values, the patients were divided into three groups : group A of 53 patients with PLT $<20 \times 109 / \mathrm{L}$; group B of 92 patients with $20 \times 109 / \mathrm{L} \leq \mathrm{PLT} \leq 30 \times 109 / \mathrm{L}$; group C of 99 patients with PLT $>30 \times 109 / \mathrm{L}$. General information including : blood cell counts, liver function, coagulation function 1 day before sugery and 1 , 7, 14 days after surgery; intraoperative blood loss ; operation time ; vital signs at the beginning, at 60 minutes and the end of the operation. Pressure and blood oxygen ; postoperative drainage ; postoperative complications and mortality.

Results : Blood cell counts, liver function, and coagulation function were improved after splenectomy in three groups $(P<0.05)$; there was no significant difference in blood loss, operation time, vital signs during the operation, postoperative drainage, postoperative complications and mortality between three groups $(P>0.05)$.

Conclusion : For HLD patients with hypersplenism, it is safe and effective to conduct splenectomy under PLT $<20 \times 10^{\circ} / \mathbf{L}$. (Acta gastroenterol. belg., 2021, 84, 51-56).
\end{abstract}

Keywords : hepatolenticular degeneration, hypersplenism, splenectomy, platelet count.

\section{Introduction}

Hepatolenticular degeneration (HLD), also known as Wilson's disease, is an autosomal recessive genetic disease with an incidence about one in 30,000 (1). Its pathogenesis lies on genetic abnormalities causing copper excretion disorders in the human body. Excess copper deposits in brain tissue, liver and kidneys, etc. Such a genetic disorder of copper metabolism inevitably results in corresponding organ lesions and dysfunction, and remains fatal if untreated (1-3). Among them, a characteristic symptom of HLD is chronic liver damage, which furtherly develops into cirrhosis and portal hypertension. Subsequently, splenomegaly and hypersplenism can occur, leading to pancytopenia, especially the platelets (PLT) (5-7). Because HLD is a hereditary disease, anti-copper drugs need to be taken for a long time or even for lifelong. At present, anti-copper drugs in clinical practice such as penicillamine and sodium dimercaptosuccinate have the side effects of inhibiting bone marrow function and causing thrombocytopenia and leukocytopenia, resulting in weakened immunity, repeated infection, and hemorrhage (8-10). Therefore, anti-copper therapy must be suspended but this will inevitably lead to the progress of the disease.
Splenectomy is a more certain and conventional treatment for hypersplenism, which can improve platelet count, white blood cell count and liver function in HLD patients with hypersplenism (11) ; and can enhance liver fibrosis and immune function in patients with hepatitis cirrhosis (12-13). HLD patients are usually accompanied with moderate or even severe platelet reduction. Even after long-time anti-copper treatment, their platelet levels still cannot recover to normal value, and patients with hyperslenism must undergo splenectomy in order to complete lifelong anti-copper therapy (11-14). Experientially, most surgeons and physicians recommend that the PLT count before splenectomy should be more than $30 \times 10^{9} / \mathrm{L}(15,16)$. However, a considerable number of patients with HLD need to perform splenectomy at low or even rare platelets level, which may increase the risk of surgery and the incidence of postoperative complications. Therefore, determining the safety range of PLT count for splenectomy is not only important for the prognosis of HLD patients with hypersplenism, but also provide a useful reference for clinical surgeons to perform splenectomy or other surgical decisions.

Previous studies on safety range of PLT count for splenectomy are focused on idiopathic thrombocytopenic purpura(17-19). Until now, to the best of our knowledge, no researches have reviewed and evaluated the safe range of PLT platelet counts for splenectomy in HLD patients with hypersplenism. Here in this work, we tried to determine the safety range of PLT count before splenectomy in HLD patients with hypersplenism by monitoring physiological parameters related to liver function and analyze the statistical data of postoperative complications and mortality.

\section{Methods}

Ethics statement

The protocol for this clinical study is fully in line with the ethical principles of the Helsinki Declaration, in line with good clinical practice guidelines and applicable local regulatory requirements. Written informed consent

Correspondence to: Qingsheng Yu, The First Affiliated Hospital of Anhui University of Chinese Medicine, Hefei, 230038, China.

E-mail :ahszyyyqs@163.com

Submission date : 08/05/2020

Acceptance date : 08/07/2020 
has been obtained from the parent or guardian of each adult or child patient. This research and written consent procedure have been approved by the Ethics Committee of the First Affiliated Hospital of Anhui University of Traditional Chinese Medicine.

\section{Patients}

This study selected 244 patients diagnosed with HLD and hypersplenism who underwent splenectomy in the General Surgery of the First Affiliated Hospital of Anhui University of Traditional Chinese Medicine from January 2012 to January 2018. All patients had moderate or severe hypersplenism and splenomegaly. The patients were divided into three groups according to their platelet value. There were 53 cases in group A with "PLT $<20 \times$ $10^{9} / \mathrm{L}$ ", 92 cases in group B with " $20 \times 10^{9} / \mathrm{L} \leq \mathrm{PLT} \leq 30 \times$ $10^{9} / \mathrm{L}$ ", and 99 cases in group C with "PLT $>30 \times 10^{9} / \mathrm{L}$ ". The included HLD patients were all of hypersplenism with cirrhosis and portal hypertension, Child-Pugh grade $\mathrm{A}$ or $\mathrm{B}$, and their kidney function was normal. While the Child-Pugh $\mathrm{C}$ patients were not recruited into surgery. Patients with cirrhosis complicated by severe jaundice, hepatic encephalopathy, refractory ascites or other advanced liver diseases, and with severe heart disease or renal failure were also excluded from surgery. All patients agreed and submitted surgical consent for possible complications.

HLD diagnostic criteria are based on clinical manifestations, positive family history, extrapyramidal symptoms or liver disease symptoms, corneal pigment ring (KF ring) positive, serum ceruloplasmin below the lower limit of normal level, 24 hours of urinary copper $>$ $100 \mu \mathrm{g}$ or elevated liver copper $>250 \mu \mathrm{g} / \mathrm{g}$ (dry weight), in accordance with the HLD diagnostic criteria formed by the 8th International Conference on Wilson Disease (20).

\section{Preoperative treatment}

Prepare routine preoperative nursing and conduct operation after all surgical requirements are met. In addition to the routine treatment of abdominal surgery, another two preparations are suggested before surgery. (1) Liver function preparation : 1) anti-copper treatment : using penicillamine and sodium dimercaptopropane sulfonate to expel copper for 6 courses (6 days for one course). 2) liver protection method : basic application of polarized liquid and branched-chain amino acid for 1 to 2 weeks before operation. Magnesium isoglycyrrhizinate, aspartate ornithine, and reduced glutathione were used as appropriate. Fresh plasma and albumin were applied if necessary. The liver function of patient reaches ChildPugh grades A and B after liver protection treatment (2). Coagulation function preparation: According to the prolonged prothrombin time and Child-Pugh classification, vitamin $\mathrm{K} 1$ and fresh plasma were supplied 1 week before surgery; prothrombin complex and platelet were used as appropriate before operation.

\section{Operative procedures}

All operative procedures were performed by same group of physicians. All patients underwent endotracheal intubation combined with intravenous anesthesia and so-called open precise splenectomy. Detailed procedure of open precise splenectomy is described as follows: open abdomen, separate gastric colon and gastric spleen ligament, exposure and ligate splenic artery, autologous spleen blood reinfusion. After that, the spleen was pulled out carefully, and the blood vessels were ligated one by one in the upper and lower ends of the spleen. The ligaments of the spleen and kidney were sharply separated, and the pancreatic tail was bluntly separated. Anterior lobe of spleen and kidney ligament was separated sharply, and then remove the spleen. The wound was excised for hemostasis and suture serosa. Finally, a drainage tube was placed in the lower part of the spleen and the abdomen was closed layer by layer.

\section{Postoperative treatment}

Routine treatment: postoperative anti-infection, fluid replacement, nutritional support, hemostasis and other symptomatic treatments. Concurrent abdominal hemorrhage treatment: postoperative monitoring the abdominal drainage and vital signs. In particular, if the drainage of blood fluid $>50 \mathrm{~mL} / \mathrm{h}$ and hemoglobin $<$ $70 \mathrm{~g} / \mathrm{L}$, then laparotomy should be conducted while other medical treatments are ineffective.

\section{Analytical assessment}

The serum was collected 1 day before splenectomy, and 1,7,14 days after splenectomy, and the level of white blood cells (WBC), red blood cells (RBC), hemoglobin (HGB), platelets (PLT), aspartate aminotransferase (AST), Alanine aminotransferase (ALT), Prothrombin time (PT), activated partial thromboplastin time (APTT), bilirubin (TBIL), International Normalized Ratio (INR), albumin (ALB), creatinine ( $\mathrm{SCr}$ ) were recorded for further analysis. Meanwhile, the Model for End-Stage Liver Disease (MELD) scores were also calculated to evaluate the liver function. The intraoperative blood loss, operation time and postoperative drainage volume were recorded. Mean arterial pressure (MAP), heart rate (HR), and blood oxygen saturation $\left(\mathrm{SPO}_{2}\right)$ were collected at the beginning of the operation $\left(\mathrm{T}_{1}\right), 60$ minutes after surgery $\left(\mathrm{T}_{2}\right)$, and at the end of surgery $\left(\mathrm{T}_{3}\right)$. The complications associated with splenectomy (abdominal hemorrhage, incisional hemorrhage, wound infection, pulmonary infection, urinary tract infection, liver failure, and fulminant infection) and mortality were recorded.

The formula for estimating intraoperative blood loss is described as(21): the total amount of liquid in the suction reservoir + (the weight of blood gauze and the net weight of gauze and normal saline) - the amount of flushing liquid. All patients with postoperative abdominal 
bleeding were diagnosed by Multi-slice spiral CT(22). we defined the deep red of drainage fluid bleeding as the severe case of abdominal bleeding, the red as the middle case of abdominal bleeding, and the light red as the mild case of abdominal bleeding.

\section{Statistical analysis}

Data analysis was performed using SPSS 23.0 (SPSS Inc, Chicago, IL, USA) statistical software. The measurement data was expressed as means \pm SD. Kolmogorov-smirnov(K-S test) was used to assess normal distribution, one-way ANOVA was used for multi-group comparison, Paired t-test was used to compare before and after treatment when the data satisfies the normal distribution, Otherwise, the Wilcoxon test was used. the count data was expressed by the number of cases and the percentage, and the disordered classification data was analyzed by $\mathrm{c}^{2}$ test, $\mathrm{P}<0.05$ was considered to be statistically significant.

\section{Results}

\section{Participants}

We analyzed 244 cases of HLD patients with hypersplenism. There were 53 cases in group A, 92 cases in group B, and 99 cases in group C. The baseline data are shown in Table 1. Compared with group $\mathrm{C}$, group $\mathrm{A}$ and group $\mathrm{B}$ had no differences in preoperative age, gender, WBC, RBC, HGB, ALT, AST, TBIL, AIB, PT, APTT, INR, SCr and MELD scores $(P>0.05)$, with the exception of PLT $(P<0.05)$.

\section{Changes of blood cell count after splenectomy}

The WBC counts of the three groups were improved compared with those before surgery $(P<0.05)$. The

Table 1. - Clinical characteristics of HLD patients with hypersplenism before splenectomy (means \pm SD)

\begin{tabular}{|c|c|c|c|c|c|}
\hline & Group A & Group B & Group C & $\mathrm{P}_{\text {value }}{ }^{\mathrm{a}}$ & $\mathrm{P}$ value \\
\hline Patients (n) & 53 & 92 & 99 & - & - \\
\hline Average age (years) & $26.68 \pm 6.93$ & $26.22 \pm 8.38$ & $28.69 \pm 9.62$ & NS & NS \\
\hline Gender $(\mathrm{M} / \mathrm{F})$ & $30 / 23$ & $49 / 43$ & $49 / 50$ & NS & NS \\
\hline $\mathrm{WBC}\left(\times 10^{9} / \mathrm{L}\right)$ & $2.28 \pm 0.83$ & $2.46 \pm 0.98$ & $2.65 \pm 1.45$ & NS & NS \\
\hline $\mathrm{RBC}\left(\times 10^{12} / \mathrm{L}\right)$ & $3.38 \pm 0.56$ & $3.25 \pm 0.49$ & $3.31 \pm 0.46$ & NS & NS \\
\hline $\operatorname{HGB}(\mathrm{g} / \mathrm{L})$ & $108.09 \pm 18.85$ & $106.41 \pm 19.30$ & $106.93 \pm 17.89$ & NS & NS \\
\hline ALT(U/L) & $38.42 \pm 17.03$ & $36.36 \pm 17.46$ & $38.80 \pm 15.99$ & NS & NS \\
\hline AST(U/L) & $41.26 \pm 16.84$ & $45.29 \pm 12.61$ & $42.13 \pm 14.67$ & NS & NS \\
\hline TBIL(mg/dl) & $1.38 \pm 0.83$ & $1.30 \pm 0.75$ & $1.19 \pm 0.61$ & NS & NS \\
\hline $\operatorname{ALB}(\mathrm{g} / \mathrm{L})$ & $37.19 \pm 4.12$ & $38.60 \pm 3.85$ & $38.79 \pm 6.04$ & NS & NS \\
\hline $\mathrm{PT}(\mathrm{s})$ & $14.64 \pm 2.47$ & $15.00 \pm 1.34$ & $15.32 \pm 2.55$ & NS & NS \\
\hline APTT(s) & $41.76 \pm 9.11$ & $43.22 \pm 7.85$ & $43.65 \pm 9.50$ & NS & NS \\
\hline INR & $1.34 \pm 0.22$ & $1.38 \pm 0.12$ & $1.40 \pm 0.22$ & NS & NS \\
\hline $\mathrm{SCr}(\mathrm{mg} / \mathrm{dl})$ & $0.87 \pm 0.14$ & $0.89 \pm 0.11$ & $0.87 \pm 0.11$ & NS & NS \\
\hline MELD scores & $8.70 \pm 3.30$ & $9.22 \pm 2.70$ & $8.92 \pm 2.96$ & NS & NS \\
\hline $\operatorname{PLT}\left(\times 10^{9} / \mathrm{L}\right)$ & $15.47 \pm 2.49$ & $24.79 \pm 2.84$ & $45.47 \pm 18.37$ & 0.000 & 0.000 \\
\hline
\end{tabular}

a : Comparison between group A and group B. b : Comparison between group A and group C. NS: no significance

Table 2. - Comparisons of blood cell counts before and after splenectomy (mean \pm SD)

\begin{tabular}{|c|c|c|c|c|c|}
\hline Time & Group & WBC $\left(10^{9} / \mathrm{L}\right)$ & $\operatorname{RBC}\left(10^{12} / \mathrm{L}\right)$ & HGB $(\mathrm{g} / \mathrm{L})$ & PLT $\left(10^{9} / \mathrm{L}\right)$ \\
\hline \multicolumn{6}{|c|}{ Preoperative } \\
\hline \multirow{3}{*}{1 day } & $\mathrm{A}$ & $2.28 \pm 0.83$ & $3.38 \pm 0.56$ & $108.09 \pm 18.85$ & $15.47 \pm 2.49$ \\
\hline & $\mathrm{B}$ & $2.46 \pm 0.98$ & $3.25 \pm 0.49$ & $106.41 \pm 19.30$ & $24.79 \pm 2.84$ \\
\hline & $\mathrm{C}$ & $2.65 \pm 1.45$ & $3.31 \pm 0.46$ & $106.93 \pm 17.89$ & $45.47 \pm 18.37$ \\
\hline \multicolumn{6}{|c|}{ Postsplenectomy } \\
\hline \multirow{3}{*}{1 day } & $\mathrm{A}$ & $17.28 \pm 5.94 *$ & $3.81 \pm 0.75^{*}$ & $113.77 \pm 9.78^{*}$ & $109.17 \pm 45.96 *$ \\
\hline & B & $17.06 \pm 6.37 *$ & $3.92 \pm 0.77 *$ & $112.35 \pm 17.01 *$ & $144.47 \pm 71.27^{*}$ \\
\hline & $\mathrm{C}$ & $15.08 \pm 4.91 *$ & $3.83 \pm 0.62 *$ & $116.28 \pm 18.38^{*}$ & $129.84 \pm 78.55^{*}$ \\
\hline \multirow{3}{*}{7 day } & $\mathrm{A}$ & $9.65 \pm 2.86^{*} \#$ & $4.16 \pm 0.70^{*} \#$ & $120.08 \pm 17.15^{* \#}$ & $295.43 \pm 148.68 * \#$ \\
\hline & $\mathrm{B}$ & $10.56 \pm 4.29 * \#$ & $4.10 \pm 0.67 * \#$ & $115.67 \pm 14.9 * \#$ & $322.51 \pm 161.09 * \#$ \\
\hline & $\mathrm{C}$ & $8.47 \pm 3.8 * \#$ & $4.04 \pm 0.69 * \#$ & $119.70 \pm 16.69 * \#$ & $322.06 \pm 161.89 * \#$ \\
\hline \multirow{3}{*}{14 day } & $\mathrm{A}$ & $7.34 \pm 2.34 * \#^{\wedge}$ & $4.66 \pm 0.62 * \#^{\wedge}$ & $125.62 \pm 12.64^{*} \#^{\wedge}$ & $420.17 \pm 133.18^{*} \#^{\wedge}$ \\
\hline & $\mathrm{B}$ & $6.77 \pm 2.11^{*} \#^{\wedge}$ & $4.82 \pm 0.53^{*} \#^{\wedge}$ & $122.74 \pm 15.03^{*} \#^{\wedge}$ & $406.39 \pm 145.7^{*} \#^{\wedge}$ \\
\hline & $\mathrm{C}$ & $5.58 \pm 1.99^{*} \#^{\wedge}$ & $4.66 \pm 0.53^{*} \#^{\wedge}$ & $124.46 \pm 13.92^{*} \#^{\wedge}$ & $428.30 \pm 159.60^{*} \#^{\wedge}$ \\
\hline
\end{tabular}

$* \mathrm{P}<0.05$, compared with presplenic splenectomy in the group ; $\mathrm{P}<0.05$, compared with 1 day after splenectomy in the group ; $\wedge \mathrm{P}<0.05$, compared with 7 days after intrasplenic splenectomy 
Table 3. - Comparison of liver function before and after splenectomy (mean \pm SD)

\begin{tabular}{|c|c|c|c|c|c|}
\hline Time & Group & ALT(U/L) & $\mathrm{AST}(\mathrm{U} / \mathrm{L})$ & $\mathrm{PT}(\mathrm{s})$ & APTT(s) \\
\hline \multicolumn{6}{|c|}{ Preoperative } \\
\hline \multirow{3}{*}{1 day } & A & $38.42 \pm 17.03$ & $41.26 \pm 16.84$ & $14.64 \pm 2.47$ & $41.76 \pm 9.11$ \\
\hline & B & $36.36 \pm 17.46$ & $45.29 \pm 12.61$ & $15.00 \pm 1.34$ & $43.22 \pm 7.85$ \\
\hline & $\mathrm{C}$ & $38.80 \pm 15.99$ & $42.13 \pm 14.67$ & $15.32 \pm 2.55$ & $43.65 \pm 9.50$ \\
\hline \multicolumn{6}{|c|}{ Postsplenectomy } \\
\hline \multirow{3}{*}{1 day } & A & $61.28 \pm 35.05^{*}$ & $63.00 \pm 31.54^{*}$ & $15.44 \pm 2.43 *$ & $45.12 \pm 10.53 *$ \\
\hline & B & $57.46 \pm 44.04 *$ & $60.12 \pm 32.11 *$ & $15.89 \pm 1.51^{*}$ & $46.40 \pm 9.82 *$ \\
\hline & $\mathrm{C}$ & $67.89 \pm 42.01 *$ & $69.35 \pm 32.99 *$ & $16.17 \pm 2.82 *$ & $48.54 \pm 12.46^{*}$ \\
\hline \multirow{3}{*}{7 day } & $\mathrm{A}$ & $46.11 \pm 25.59^{*}$ & $45.11 \pm 15.96^{\#}$ & $14.71 \pm 2.32^{\#}$ & $39.85 \pm 9.66^{\#}$ \\
\hline & B & $42.85 \pm 27.76^{\#}$ & $41.03 \pm 15.59^{\#}$ & $14.96 \pm 1.19^{*}$ & $40.23 \pm 10.68^{\sharp}$ \\
\hline & $\mathrm{C}$ & $48.58 \pm 29.47^{\#}$ & $43.62 \pm 20.06^{H}$ & $15.02 \pm 2.34^{\#}$ & $41.53 \pm 10.93^{\#}$ \\
\hline \multirow{3}{*}{14 day } & A & $28.13 \pm 10.43^{* \# \wedge}$ & $32.38 \pm 12.91^{* \# \wedge}$ & $13.69 \pm 2.10^{* \# \wedge}$ & $34.42 \pm 7.26^{* \# \wedge}$ \\
\hline & $\mathrm{B}$ & $27.15 \pm 14.74^{* * \wedge}$ & $34.46 \pm 18.01^{* \# \wedge}$ & $13.88 \pm 1.50^{* \# \wedge}$ & $36.73 \pm 10.86^{* \# \wedge}$ \\
\hline & $\mathrm{C}$ & $30.96 \pm 14.12^{* * \wedge}$ & $33.91 \pm 13.66^{* * \wedge}$ & $13.68 \pm 2.45^{* * \wedge}$ & $35.80 \pm 7.57^{* \# \wedge}$ \\
\hline
\end{tabular}

* $\mathrm{P}<0.05$, compared with before splenectomy in the group ; $\mathrm{P}<0.05$, compared with 1 day after splenectomy in the group ; ${ }^{\wedge} \mathrm{P}<0.05$, compared with 7 days after intrasplenic splenectomy.

Table 4. - Comparison of splenectomy results and postoperative details (mean \pm SD)

\begin{tabular}{|c|c|c|c|c|c|c|}
\hline Variables & Time & Group A & Group B & Group C & $P$ value ${ }^{\mathrm{a}}$ & $P$ value ${ }^{b}$ \\
\hline Intraoperative blood loss (ml) & $\mathrm{T}_{1}-\mathrm{T}_{3}$ & $137.08 \pm 93.4$ & $139.73 \pm 104.98$ & $171.72 \pm 141.27$ & NS & NS \\
\hline Operation time $(\mathrm{min})$ & $\mathrm{T}_{1}-\mathrm{T}_{3}$ & $104.45 \pm 15.08$ & $106.65 \pm 17.06$ & $108.36 \pm 17.39$ & NS & NS \\
\hline \multirow{3}{*}{ MAP (mmHg) } & $\mathrm{T}_{1}$ & $89.84 \pm 10.99$ & $88.42 \pm 10.99$ & $87.96 \pm 11.25$ & NS & NS \\
\hline & $\mathrm{T}_{2}$ & $89.17 \pm 12.14$ & $87.47 \pm 9.75$ & $87.83 \pm 10.73$ & NS & NS \\
\hline & $\mathrm{T}_{3}$ & $88.70 \pm 11.37$ & $88.99 \pm 10.47$ & $86.62 \pm 10.03$ & NS & NS \\
\hline \multirow{3}{*}{ HR (beats/min) } & $\mathrm{T}_{1}$ & $80.21 \pm 9.13$ & $80.66 \pm 8.30$ & $81.39 \pm 8.21$ & NS & NS \\
\hline & $\mathrm{T}_{2}$ & $81.47 \pm 8.89$ & $79.64 \pm 8.36$ & $81.91 \pm 9.23$ & NS & NS \\
\hline & $\mathrm{T}_{3}$ & $81.36 \pm 8.49$ & $80.71 \pm 8.07$ & $82.89 \pm 7.69$ & NS & NS \\
\hline \multirow{3}{*}{$\mathrm{SPO}_{2}(\%)$} & $\mathrm{T}_{1}$ & $98.43 \pm 1.03$ & $98.28 \pm 0.92$ & $98.41 \pm 1.05$ & NS & NS \\
\hline & $\mathrm{T}_{2}$ & $98.43 \pm 1.10$ & $98.26 \pm 0.95$ & $98.35 \pm 0.95$ & NS & NS \\
\hline & $\mathrm{T}_{3}$ & $98.42 \pm 1.17$ & $98.53 \pm 0.92$ & $98.33 \pm 0.94$ & NS & NS \\
\hline Postoperative drainage $(\mathrm{ml})$ & - & $90.05 \pm 45.69$ & $102.09 \pm 65.54$ & $96.86 \pm 68.96$ & NS & NS \\
\hline
\end{tabular}

$\mathrm{a}$ :Comparison between group A and group B. $\mathrm{b}$ :Comparison between group A and group C. NS : no significance

WBC counts reached the highest on the first day after surgery, then decreased gradually on the 7th and 14th days after operation and recovered to the normal level. The RBC, HGB, and PLT counts in the three groups were higher than those before surgery $(P<0.05)$ at 1 day, 7 days, and 14 days, and showed a tendency of gradual increase. Detailed data was shown in Table 2.

\section{Changes of liver function and coagulation function after splenectomy}

As shown in Table 3, the ALT, AST, PT and APTT level in three groups were all increased on the $1 \mathrm{st}$ day after splenectomy $(P<0.05)$, and then gradually decreased on the 7 th and 14 th day after splenectomy $(P$ $<0.05)$.

Comparison of surgical details during and after splenectomy

Compared with group $\mathrm{C}$, the operation time in group A and group B was longer, with slightly increased blood loss and postoperative drainage fluid volume, but the difference was not statistically significant $(P>0.05)$.
Comparison of vital signs such as MAP, HR, and $\mathrm{SPO}_{2}$ in three groups $\left(\mathrm{T}_{1}, \mathrm{~T}_{2}\right.$, and $\left.\mathrm{T}_{3}\right)$ also showed no statistical difference between three groups $(P>0.05)$. See Table 4 .

\section{Comparison of postoperative complications and mortality-}

The comparison of postoperative complications and mortality after splenectomy in three groups were recorded in Table 5. Without a single case of death, complications occurred in 30 patients $(12.3 \%)$ in three groups. Among them, both group B and group C had one serious adverse reaction of fulminant infection, and they were cured under early detection and active treatment. All other complications in three groups were also cured after conservative treatment. Importantly, there was no statistical difference in the incidence of postoperative complications between three groups $(P>0.05)$.

\section{Discussion}

In the present work, we noticed that by comparing three groups of HLD patients with different PLT level 
Table 5. - Comparison of complications after splenectomy (n)

\begin{tabular}{|c|c|c|c|c|c|}
\hline Variables & Group A & Group B & Group C & $P$ value ${ }^{\mathrm{a}}$ & $P$ value ${ }^{\mathrm{b}}$ \\
\hline The severe case of abdominal hemorrhage & - & - & - & & \\
\hline The middle case of abdominal hemorrhage & - & 1 & 1 & & \\
\hline Incision bleeding & 1 & 2 & 1 & & \\
\hline Incision infection & 1 & 2 & 4 & & \\
\hline lung infection & 1 & 1 & 2 & & \\
\hline Acute liver failure & - & - & - & & \\
\hline Fulminant infection & - & 1 & 1 & & \\
\hline Total & $7(13.2 \%)$ & $10(10.9 \%)$ & $13(13.1 \%)$ & NS & NS \\
\hline
\end{tabular}

a : Comparison between group A and group B. b : Comparison between group A and group C. NS : no significance

who underwent splenectomy, there was no significant difference in the amount of bleeding and the vital signs (MAP, HR, $\mathrm{SPO}_{2}$ ) during the operation $\left(\mathrm{T}_{1}, \mathrm{~T}_{2}\right.$, $\mathrm{T}_{3}$ ). Besides, the blood cells count (RBC, WBC, HGB) in each group significantly increased after operation, proving the efficacy of splenectomy. Therefore, we emphasize that splenectomy is safe and effective for hypersplenism in HLD patients with PLT lower than $20 \times 10^{9} / \mathrm{L}$, which can increase blood cells amount and stabilize vital signs of patients without risking intraoperative and postoperative bleeding. Furthermore, there is no significant difference in the morbidity and type of complications (abdominal hemorrhage, incision bleeding, incision infection, lung infection, urinary system infection, acute liver failure, fulminant infection) between three groups. As for mortality, not a single case of death was found in three groups. Based on 244 cases of splenectomy for hypersplenism in HLD patients with different PLT level, a conclusion can be made that splenectomy is safe and effective for HLD patients with PLT lower than $20 \times 10^{9} / \mathrm{L}$.

The coagulation mechanism is not only composed of PLT, liver function also plays an important role through the synthesis of 4 coagulation factors. (23-24). In our work, we found that splenectomy can improve both liver function and coagulation function in HLD patients. In addition, it is worthwhile mentioning that liver function and coagulation function of three groups were normal or close to normal before surgery due to our careful preoperative treatment. Such a phenomenon suggested that even if PLT level is low, splenectomy can still be performed under clinical requirement as long as liver function and coagulation mechanism are normal or close to normal before surgery. However, if PLT level is extremely low or liver function and coagulation function are severely abnormal, we do not advocate surgery. Under this condition, long term splenic embolization interventional surgery is suggested to enhance liver function by copper-excretion and simultaneously improve coagulation mechanism. Then corresponding surgical strategy should be decided depending on the state of improved liver function and coagulation mechanism. Moreover, it should be noted that low PLT level do not inevitably increase the risk of intraoperative blood loss and postoperative complications. We speculate that the coagulation function of PLT is not only related to the quantity, but also related to the quality. It is generally week and aging platelets that are devoured by sthenic spleen.

According to our surgical experience, splenectomy can be conducted even at low PLT level, but we must highlight that careful preoperative treatment, precise operation during surgery and meticulous treatment after surgery are essential prerequisites for a successful operation and prognosis : (1) 5 to 7 days before surgery, conventional liver protection treatment should be conducted such as applying VitK1; prothrombin complex and fresh frozen plasma can be given as appropriate half an hour before surgery. (2) Surgery should be conducted under good anesthesia and exposure, with careful and delicate operating procedure. Isolation, cutting, and ligation should be performed under direct vision. Meanwhile, the splenic artery should be ligated first to reinfuse the spleen blood and reduce blood transfusion. (3) For unmanageable bleeding during operation, we realize that the lack of coagulation factors is one of the primary cause. Repeating surgical intervention is strongly objected under this condition. In fact, relying solely on electrocautery and suture hemostasis usually shows no effect or even induces deterioration. Right treatment at this time depends on the effective correction of intraoperative coagulopathy and reasonable surgical interventions. The wound can be covered by hemostatic gauze or gelatin sponge, and compressed by dry gauze to slow the bleeding. Simultaneously, Coagulation function can be corrected by : 1) quickly infuse $600-800 \mathrm{ml}$ fresh frozen plasma in a short period; 2) supplement of coagulation factors : prothrombin complex containing II, VII, IX factors are preferred ; 3) the application of calcium ; 4) the application of vitamin $C$; 5) the application of hemostatic drugs.

\section{Conclusions}

In summary, based on 244 cases of splenectomy for hypersplenism in HLD patients with different level of 
PLT, we conclude that it is safe and effective to perform splenectomy for patients with PLT lower than $20 \times$ $10^{9} / \mathrm{L}$ under careful preoperative preparation and precise operation. The result of this study may provide valuable reference for splenectomy caused by hypersplenism or other pathogenesis. This study also needs a multicenter, reasonable, randomized double-blind controlled clinical trial with sufficient sample size to further confirm our conclusion.

\section{Conflicts of interest}

All authors declare that there is no conflict of interest.

\section{Data statement}

All data included in this study are available upon request by contacting with the corresponding author (E-mail : ahszyyfh@163.com).

\section{Declaration of interest}

None.

\section{Funding}

Supported by the grants from 12th Five-Year national key clinical specialist construction project ([2013] 239).

\section{Author contribution}

*Shitang Wang ,Hui Feng and Hui Peng contributed equally for the manuscript, and should be as Joint first authors.

Qingsheng Yu, Shitang Wang and Hui Peng contributed to study conception and design, Long Huang and Fei Hui contributed to acquisition, analysis and interpretation of data, Shitang Wang contributed to drafting of manuscript, Fuhai Zhou contributed to critical revision.

\section{Acknowledgements}

The authors are deeply indebted to Zhou Zheng for their support in statistics and methodology.

\section{References}

1. KATHAWALA M, GM HIRSCHFIELD. Insights into the management of Wilson's disease. Therap. Adv. Gastroenterol., 2017, 10(11) : 889-905.

2. FERENCI P. Diagnosis of Wilson disease. Handb. Clin. Neurol., 2017,142 171-180.

3. ROBERTS EA. Update on the Diagnosis and Management of Wilson Disease. Curr. Gastroenterol. Rep., 2018, 20(12) : 56.
4. CLEYMAET S, NAGAYOSHI K, GETTINGS E, FADEN J. A review and update on the diagnosis and treatment of neuropsychiatric Wilson disease. Expert Review of Neurotherapeutics, 2019,19(11) : 1117-1126.

5. BOYER TD, HABIB S. Big spleens and hypersplenism : fix it or forget it? Liver Int., 2015, 35(5) : 1492-1498.

6. XIAOXI P, TENGYU L, WANG CHENG'EN. Splenic artery embolization with detachable balloons for hypersplenism. Journal of International Medical Research, 2018, 46(10) : 4111-4119.

7. WANG P, LI Z, REN S, LI J, YANG J, KONG G, et al. Spleen Regulates Hematopoietic Stem/Progenitor Cell Functions Through Regulation of EGF in Cirrhotic Hypersplenism. Digestive diseases and sciences, 2018, 63(7) : 1860-1867.

8. CAI S, GONG JY, YANG J, WANG JS. Anemia following zinc treatment for Wilson's disease : a case report and literature review. BMC Gastroenterol., 2019, 19(1) : 120.

9. GUPTA P, M CHOKSI, A GOEL, ZACHARIAH U, SAJITH KG, RAMACHANDRAN J. Maintenance zinc therapy after initial penicillamine chelation to treat symptomatic hepatic Wilson's disease in resource constrained setting. Indian J. Gastroenterol., 2018, 37(1) : 31-38.

10. LIWJ, CHEN C, YOU ZF, YANG RM, WANG XP. Current Drug Managements of Wilson's Disease : From West to East. Curr. Neuropharmacol., 2016, 14(4) : 322-325.

11. LI LY, YANG WM, CHEN HZ, WU YH, FANG X, ZHANG J, et al. Successful Splenectomy for Hypersplenism in Wilson's Disease : A Single Center Experience from China. PLoS One, 2015,10(4) : e0124569.

12. ZENG DB, DI L, ZHANG RC, GUO QL, DUAN BW, JIA CY, et al. The Effect of Splenectomy on the Reversal of Cirrhosis : a Prospective Study. Gastroenterol. Res. Pract., 2019, 2019 : 5459427.

13. HIRAKAWA Y, OGATA T, SASADA T, YAMASHITA T, ITOH $\mathrm{K}$, TANAKA $\mathrm{H}$, et al. Immunological consequences following splenectomy in patients with liver cirrhosis. Exp. Ther. Med., 2019, 18(1) : 848-856.

14. OWEN CA, GOLDSTEIN NP, BOWIE JW. Platelet function and coagulation in patients with Wilson disease. Archives of Internal Medicine, 1976, 136(2) : 148-152.

15. HABERMALZ B, SAUERLAND S, DECKER G, DELAITRE B, GIGOT JF, LEANDROS E, et al. Laparoscopic splenectomy : the clinical practice guidelines of the European Association for Endoscopic Surgery (EAES). Surgical Endoscopy, 2008, 22(4) : 821-848.

16. KEIDAR A, SAGI B, SZOLD A. Laparoscopic splenectomy for immune thrombocytopenic purpura in patients with severe refractory thrombocytopenia. Pathophysiol. Haemost. Thromb., 2003. 33(2) : 116-119.

17. CHEN X, PENG B, CAI Y, ZHOU J, WANG Y, WU Z, et al. Laparoscopic splenectomy for patients with immune thrombocytopenia and very low platelet count : is platelet transfusion necessary? J. Surg. Res., 2011, 170(2) : 225-232.

18. CAI Y, LIU X, PENG B. Should we routinely transfuse platelet for immune thrombocytopenia patients with platelet count less than $10 \times 10(9) / \mathrm{L}$ who underwent laparoscopic splenectomy? World J. Surg., 2014, 38(9) : 22672272.

19. WU Z, ZHOU J, LI J, ZHU Y, PENG B. The feasibility of laparoscopic splenectomy for ITP patients without preoperative platelet transfusion. Hepatogastroenterology, 2012, 59(113) : 81-85.

20. FERENCI P, CACA K, LOUDIANOS G, MIELI-VERGANI G, TANNER $\mathrm{S}$, STERNLIEB I, et al. Diagnosis and phenotypic classification of Wilson disease. Liver Int., 2003. 23(3) : 139-142.

21. ZHANG X, YU X, HUANG Y. The Correlation of Indices in r-TEG with Intra-operative Blood Loss in Neurosurgical Patients. Chin. Med. Sci. J., 2017, 32(2) : 69-74.

22. TASU JP, VESSELLE G, HERPE G, FERRIE JC, CHAN P, BOUCEBCI S, et al. Postoperative abdominal bleeding. Diagn. Interv. Imaging, 2015, 96(78) : 823-831.

23. O"CONNOR SD, TAYLOR AJ, WILLIAMS EC, WINTER TC. Coagulation Concepts Update. American Journal of Roentgenology, 2009, 193(6) : 16561664.

24. PIETERS M, WOLBERG AS. Fibrinogen and fibrin : An illustrated review. Res. Pract. Thromb. Haemost., 2019, 3(2) : 161-172. 